\title{
HMGN5 blockade by siRNA enhances apoptosis, suppresses invasion and increases chemosensitivity to temozolomide in meningiomas
}

\author{
JING HE, CHAOYANG LIU, BIN WANG, NA LI, GUOQIN ZUO and DEWEI GAO \\ Integrated Surgery Department of South Building of Chinese PLA General Hospital, Beijing 100853, P.R. China
}

Received June 20, 2015; Accepted August 6, 2015

DOI: 10.3892/ijo.2015.3131

\begin{abstract}
The high-mobility group nucleosome-binding protein-5 (HMGN5) is frequently overexpressed in various malignant cancers. However, the potential correlation between HMGN5 and prognosis in patients with meningiomas remains unknown. In the present study, we explored the expression of HMGN5 in meningiomas with immunohistochemistry and correlated the results to the patient outcome. Potential effects of HMGN5 on tumor growth, apoptosis and invasion were also examined in representative cell lines (IOMM-Lee and CH157) by downregulating HMGN5 with RNA interference (siRNA). We demonstrate that there is a positive association between HMGN5 expression and meningioma histological grade. Statistical analysis reveals that lower HMGN5 expression predict lower meningioma recurrence. In addition, downregulation of HMGN5 inhibits IOMM-Lee and CH157 cell proliferation, enhances cell apoptosis and suppresses tumor invasion. Our results further revealed that HMGN5 inhibition decreased P-glycoprotein (MDR-1) expression without affecting multidrug resistance associated proteins 1 (MRP-1) expression to increase chemosensitivity to temozolomide (TMZ) of meningioma cells. Collectively, this study indicates that HMGN5 is a novel target for developing effective therapeutic strategies for malignant meningiomas.
\end{abstract}

\section{Introduction}

Meningiomas represent the most common benign brain tumors in adults, with an annual incidence of $\sim 0.0013-0.0078 \%$ (1). It accounts for $13-26 \%$ of primary intracranial tumors and mainly classified into three histology subtypes: classical (WHO I, $>90 \%$ ), atypical (WHO II, 5-7\%) and anaplastic (WHO III, $1-3 \%$ ) variants (2). Despite a large majority being classified as benign lesions, the clinical implementation for aggressive meningiomas (WHO II, III) yield depressing results and

Correspondence to: Dr Dewei Gao, Integrated Surgery Department of South Building of Chinese PLA General Hospital, 28 Fuxing Road, Haidian District, Beijing 100853, P.R. China

E-mail: gaodewei@hotmail.com

Key words: HMGN5, meningiomas, apoptosis, invasion, temozolomide patients with malignant meningiomas rarely achieve cure $(3,4)$. Therefore, it is urgent to identify new molecular biomarkers that regulate malignant behavior of meninigomas and predict clinical outcome of patients with high-grade meninigomas.

High-mobility group nucleosome-binding proteins (HMGN/NSBP) are a family of ubiquitous nuclear proteins which participate in various physiological process, including DNA repair, replication, transcription and recombination (5). HMGN5, a typical member of the HMGN family, can modulate the cellular epigenetic profile and impact biological activities (5-7). Recently, aberrant high expression level of HMGN5 has been found in several malignant tumors, including bladder, prostate, renal cancers and glioma (8-11). Importantly, there is a positive association between excessive expression of HMGN5 and poor clinical outcomes in patients with glioma (11), suggesting that increased HMGN5 level may be critical to patient survival. Furthermore, it has been reported that HMGN5 plays an oncogenic role in clear cell renal cell carcinomas by promoting cell proliferation and invasion, and could be utilized as a target for cancer treatment (8). However, its expression pattern, biological function, and clinical significance in meningiomas are still unknown.

In this study, we show that HMGN5 overexpression is correlated with advanced pathological grade and poorer prognosis. Specially, we decreased HMGN5 expression in IOMM-Lee and $\mathrm{CH} 157$ cells by small interfering RNA (siRNA) to clarify the role of HMGN5 on cell apoptosis, proliferation and invasion in vitro. Moreover, the effects of knocking down HMGN5 expression on chemosensitivity to temozolomide (TMZ) in IOMM-Lee and CH157 meningioma cancer cells were also assessed. We believe HMGN5 could be a novel molecular target for meninigioma therapy in future.

\section{Materials and methods}

Tissue samples. The meningioma specimens were obtained with the approval from the Specialty Committee on Ethics of Biomedicine Research, PLA General Hospital. Tissue specimens were collected from 102 patients with complete clinical and follow-up information who underwent surgery from August 2004 to July 2011 in PLA General Hospital. The clinical characteristics of the patient cohort are summarized in Table I. The selection criteria were as follows: i) complete clinical data; ii) the subject had a diagnosis of meningioma 
without history of other tumors; ii) complete clinical data; iii) the subject underwent evaluation by enhanced head MRI scans for tumor progression after surgery at least once every six months.

Cell culture and transfection. The meningioma cell lines IOMM-Lee, CH157 and Ben-Men-1 were obtained from the Chinese Academy of Sciences (Shanghai, China). The three cell lines were grown in MEM medium with $10 \%$ fetal bovine serum and maintained in monolayer culture at $37^{\circ} \mathrm{C}$ in humidified air with $5 \% \mathrm{CO}_{2}$. Cells were transfected with small interfering RNA (siRNA) that targets HMGN5 (HMGN5 siRNA, Sigma-Aldrich, NM_030763) according to the manufacturer's protocols. Three different synthetic interfering RNA (siRNA) sequences were tested for inhibitory activity against HMGN5 expression by transient transfection into HEK293 cells. The most effective sequence was cloned into the pLVTHM vector. This short hairpin sequence specific for HMGN5 is 5'-GCAGTTGCTGAAACCAAGC-3'. Green fluorescent protein (GFP) was used to create non-targeting GFP-siRNA. Conditioned medium containing lentiviruses was harvested $48 \mathrm{~h}$ after transfection of HEK 293 cells. This medium was filtered and used to infect recipient cells in the presence of $8 \mu \mathrm{g} / \mathrm{ml}$ polybrene. After 2-3 weeks, single independent clones were randomly isolated and each individual clone was plated separately. After clonal expansion, cells from each independent clone were tested for HMGN5 expression by immunoblotting.

Immunohistochemistry and expression analysis. All sections were incubated in non-immune serum at $4^{\circ} \mathrm{C}$ overnight in HMGN5 antibody (1:500; Sigma-Aldrich, HPA000511). The positive percentage and the intensity of staining of cells were divided as previously described (12). The final score of HMGN5 expression was the product of the HMGN5 expression rate and intensity $(-, 0 ;+, 1-3 ;++, 4-6 ;++, 7-9)$. For statistical analysis, HMGN5 expression was divided into 'high' $(++$ and +++$)$ vs. 'low' (+ and -).

Cell proliferation assay. Cell viability was determined by the 3-(4,5-dimethylthiazol-2-yl)-2,5-diphenyltetrazolium bromide (MTT) assay. MTT (5 mg/ml) was added to cells for $4 \mathrm{~h}$ at $37^{\circ} \mathrm{C}$. Dimethyl sulfoxide (DMSO) was used to dissolve the formazan crystals produced from MTT by live cells. The microplate reader (Thermo Scientific) was used to measure the optical density at $570 \mathrm{~nm}$.

Quantitative RT-PCR analysis. The quantitative RT-PCR analysis for mRNA level was performed as previously described (12). The PCR were conducted as follows: $5 \mathrm{~min}$ at $95^{\circ} \mathrm{C}$ followed by 40 cycles of $94^{\circ} \mathrm{C}$ for $30 \mathrm{sec}, 50^{\circ} \mathrm{C}$ for $30 \mathrm{sec}$ and $72^{\circ} \mathrm{C}$ for $30 \mathrm{sec}$. The primers for HMGN5, MMP-2, Bcl-2, Bcl-2 (B-cell lymphoma-2), caspase-3, and $\beta$-actin were designed as follows: HMGN5, forward, 5'-GGTTGTCTG CTATGCTTGTG-3'; reverse, 5'-ACTGCTTCTTGCTTGGT TTC-3'. MMP-2, forward, 5'-AGATCTTCTTCTTCAAGGAC CGGTT-3';reverse,5'-GGCTGGTCAGTGGCTTGGGGTA-3'. Bcl-2, forward, 5'-CCGGGAGATCGTGATGAAGT-3'; reverse, 5'-ATCCCAGCCTCCGTTATCCT-3'. Caspase-3, forward, 5'-ATGGAGAACAATAAAACCT-3'; reverse, 5'-CTAGTGAT AAAAGTAGAGTTC-3'. $\beta$-actin, forward, 5'-TGACGTGGAC
Table I. Correlation between HMGN5 immunoreactivity and clinicopathologic characteristics of meningioma patients.

\begin{tabular}{|c|c|c|c|c|}
\hline \multirow[b]{2}{*}{ Characteristics } & \multirow[b]{2}{*}{ Value } & \multicolumn{2}{|c|}{ HMGN5 expression } & \multirow[b]{2}{*}{ P-value } \\
\hline & & Low & High & \\
\hline No. of patients & 102 & 57 & 45 & \\
\hline Age, years & & & & 0.12 \\
\hline$<60$ & 68 & 40 & 28 & \\
\hline$\geq 60$ & 34 & 17 & 17 & \\
\hline Gender & & & & 0.15 \\
\hline Male & 36 & 19 & 17 & \\
\hline Female & 66 & 38 & 28 & \\
\hline Tumor location & & & & 0.18 \\
\hline Convexity & 32 & 16 & 16 & \\
\hline Parasagittal sinus & 25 & 11 & 14 & \\
\hline Parafalcine & 20 & 12 & 8 & 0.23 \\
\hline Skull base & 25 & 18 & 7 & \\
\hline Tumor size & & & & 0.17 \\
\hline$<3 \mathrm{~cm}$ & 29 & 16 & 13 & \\
\hline$\geq 3 \mathrm{~cm}$ & 73 & 41 & 32 & \\
\hline Extent of resection & & & & 0.11 \\
\hline Simpson grade I & 49 & 26 & 23 & \\
\hline Simpson grade II & 40 & 23 & 17 & \\
\hline Simpson grade III & 13 & 8 & 5 & \\
\hline Histological grade & & & & 0.008 \\
\hline I (classical) & 70 & 45 & 25 & \\
\hline II (atypical) & 18 & 10 & 8 & \\
\hline III (anaplastic) & 14 & 2 & 12 & \\
\hline Recurrence & & & & 0.004 \\
\hline Negative & 74 & 50 & 24 & \\
\hline Positive & 28 & 7 & 21 & \\
\hline
\end{tabular}

ATCCGCAAAG-3'; reverse 5'-CTGGA AGGTGGACAGCG AGG-3'.

Protein extraction and western blotting. Western blot analysis was conducted as described previously. All operations were completed on ice at $4^{\circ} \mathrm{C}$. The primary antibodies used were HMGN5 (Sigma-Aldrich, SAB1305759, 1:1,000), Bcl-2 (Sigma-Aldrich, 1:500), caspase-3 (Santa Cruz, 1:1,000), and $\beta$-actin (Santa Cruz, 1:1,000). Western blot data were quantified by normalizing the signal intensity of each sample to that of $\beta$-actin.

Invasion assay. Equal number of untransfected, HMGN5siRNA-transfected and GFP-siRNA-transfected cells were plated onto a 24-well culture plate. Adherent cells in the upper surface of the filter were removed, and the cells on the lower surface were fixed with $3.7 \%$ formaldehyde. The cells were stained with hematoxylin and counted. Three independent experiments were determined for the invasion rate.

Apoptosis assay. Annexin V-FITC apoptosis kit was used to measure apoptosis according to the manufacturer's instruc- 
A

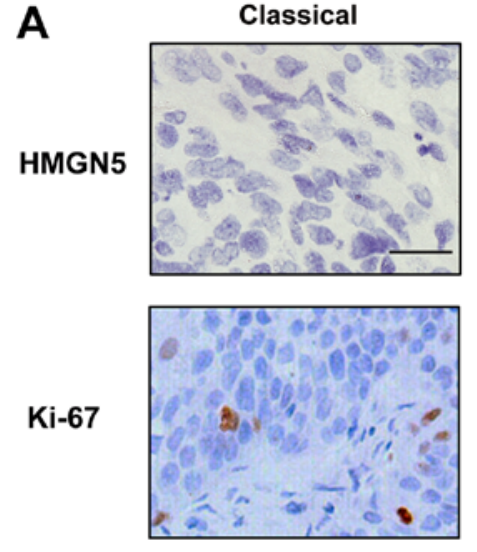

B

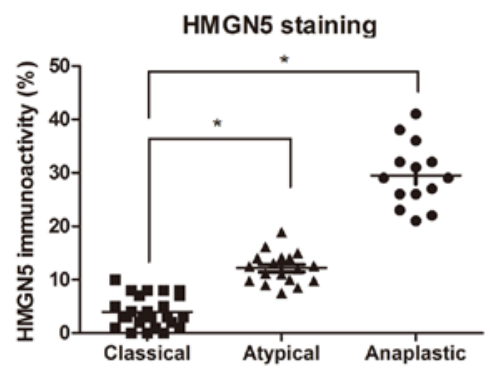

D

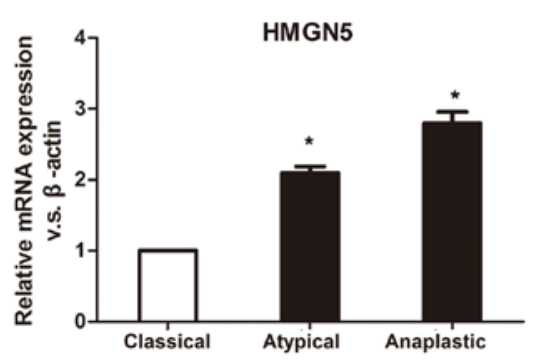

$\mathbf{F}$

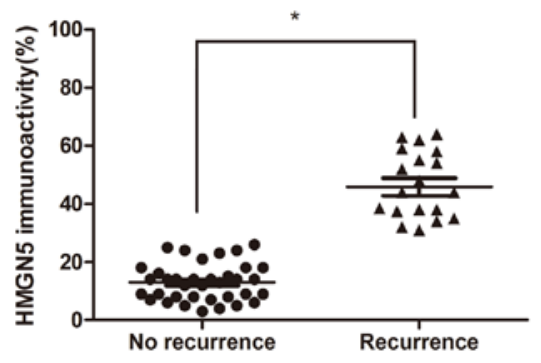

Atypical
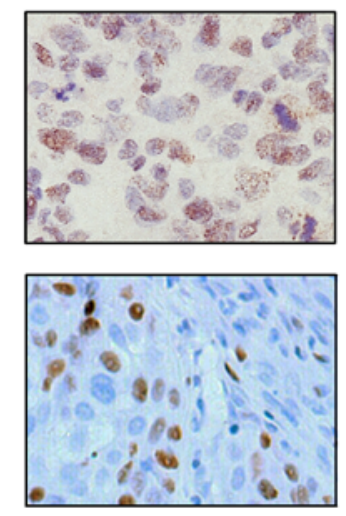

Anaplastic
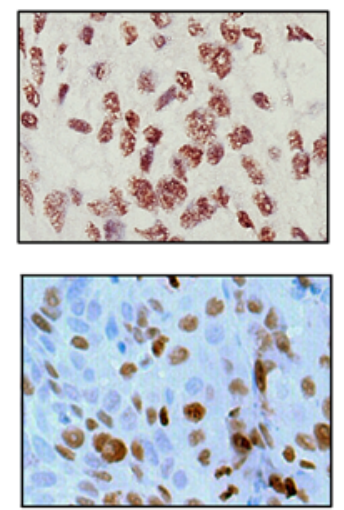

C

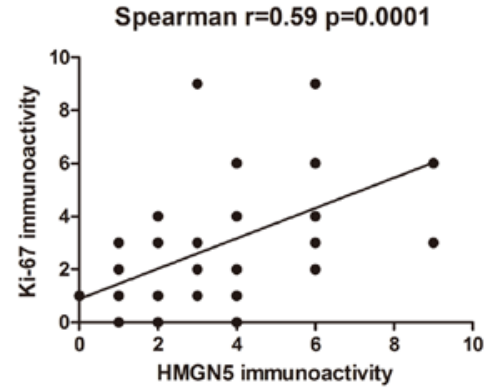

E

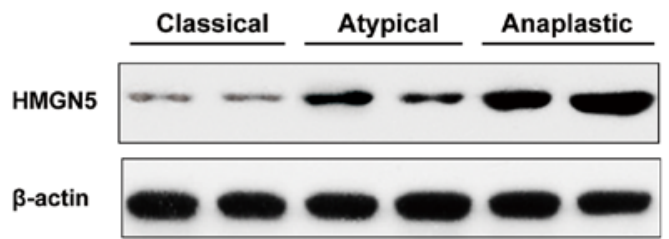

G

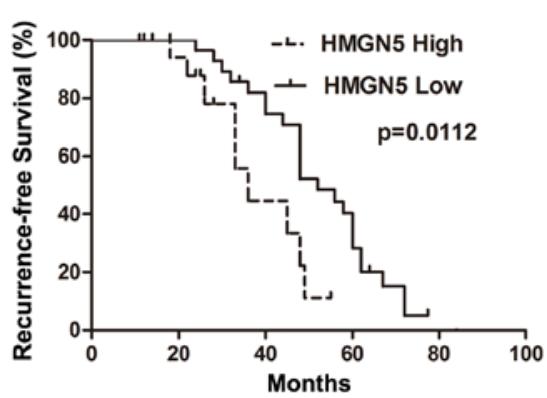

Figure 1. (A) Immunohistochemical images of meningiomas for HMGN5 and Ki-67. Bar, $20 \mu \mathrm{m}$. (B) The quantification of HMGN5 expression was expressed by percentage of HMGN5-immunopositive cells. The HMGN5 expression level was correlated with increased pathological grade (P=0.008). (C) Significant association between HMGN5 and Ki-67 immunostaining scores suggested that HMGN5 expression is correlated with high proliferation rate. (D) mRNA levels of HMGN5 measured in meningiomas by qRT-PCR. ${ }^{*} \mathrm{P}<0.05$. (E) Expression of HMGN5 proteins in classical, atypical and anaplastic meningiomas measured by western blotting. (F) The dot blot for HMGN5 expression in meningiomas revealed that the tumors with recurrence had high HMGN5 expression. P=0.004 (G) Kaplan-Meier survival curves according to HMGN5 expression. $\mathrm{P}=0.0112$.

tions (Immunochemistry Technologies). For each experiment, $\geq 20,000$ cells were analyzed.

Statistical analyses. The data are expressed as mean \pm SD for triplicate determination, and analyzed using Student's t-test. Kaplan-Meier analysis was used to investigate overall survival (OS) in meningioma patients. SPSS 16.0 was used to conduct analyses and $\mathrm{P}<0.05$ was considered statistically significant.

\section{Results}

HMGN5 is overexpressed in human meningiomas and correlates with poor prognosis. We first used immunohistochemical staining to measure the expression levels of HMGN5 and Ki-67 in 102 human meningioma specimens. Advanced meningiomas (WHO grade II and III) showed greater immunoreactivity for HMGN5 and Ki-67 expressions compared to classical meningiomas (WHO grade I) (Fig. 1A) and the 
A

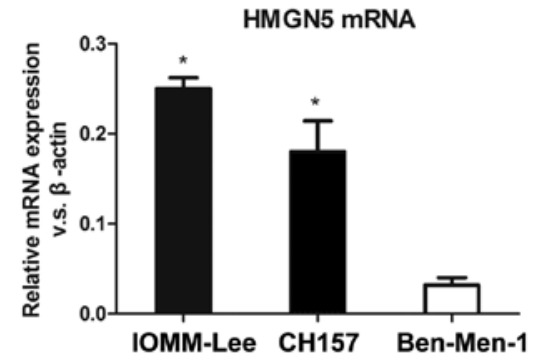

C

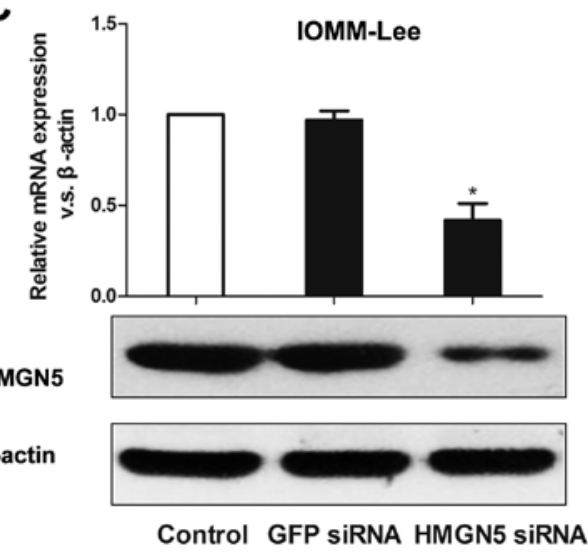

B IOMM-Lee CH157 Ben-Men-1

HMGN5

$\beta$-actin

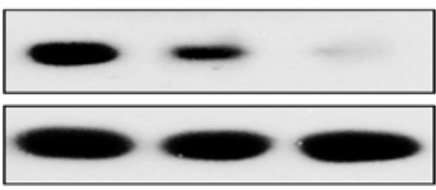

D

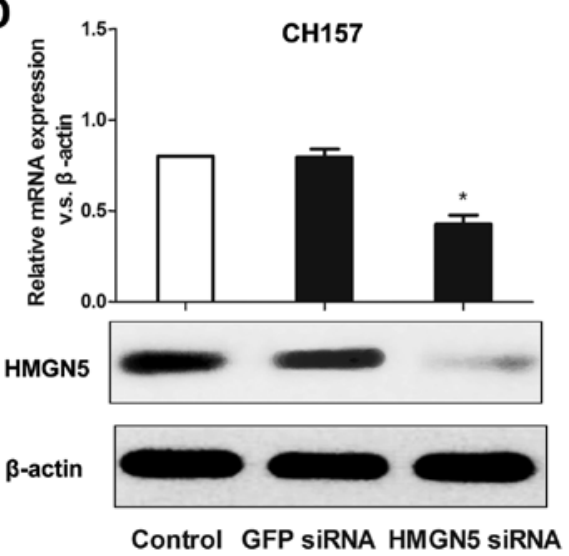

Figure 2. (A) mRNA levels of HMGN5 measured in Ben-Men-1, IOMM-Lee, and CH157 cells by qRT-PCR. (B) Western blotting revealed similar results in the three meningioma cell lines. qRT-PCR and western blotting showed that the expression of HMGN5 was significantly decreased in IOMM-Lee (C) and CH157 (D) cells transfected with HMGN5-targeted siRNA on mRNA and protein levels.

expression level of HMGN5 correlated with the histological grading $(\mathrm{P}=0.008)$ (Fig. 1B). Statistical analyses indicated that HMGN5 immunolabeling significantly associated with immunolabeling for Ki-67 (P=0.0001) (Fig. 1C). Consistent with immunostaining results, atypical and anaplastic meningiomas exhibited dramatically higher HMGN5 mRNA expression than classical meningiomas (Fig. 1D). Western blotting confirmed the similar results (Fig. 1E). In addition, despite the benign tumors having low HMGN5 expression, aberrant high levels of HMGN5 expression was observed in meningiomas with recurrence (Fig. 1F), suggesting that HMGN5 expression may induce more aggressive tumor behavior.

The demographic and clinicopathological characteristics stratified by high HMGN5 and low HMGN5 are summarized in Table I. We showed that HMGN5 expression was positively correlated with histological grade $(\mathrm{P}=0.008)$, and recurrence $(\mathrm{P}=0.004)$. However, no significant associations were found between HMGN5 expression and other clinicopathological features (Table I). In addition, Kaplan-Meier analysis revealed that patients in low HMGN5 expression group had significantly longer recurrence-free survival than those in high HMGN5 expression group $(\mathrm{P}=0.0112)$ (Fig. 1G). These results suggested that HMGN5 might be a critical molecular biomarker that predicts the recurrence of patients with meningiomas.

Altered HMGN5 expression affects proliferation and apoptosis in meningiomas. We first measured HMGN5 expression in three meningioma cell lines (Ben-Men-1, IOMM-Lee, and CH157) by using RT-PCR. The mRNA expression levels of HMGN5 normalized by $\beta$-actin mRNA in IOMM-Lee,
CH157, and Ben-Men-1 cells were 0.2538 $\pm 0.0162(\mathrm{P}=0.0002$ vs. Ben-Men-1), 0.1824 \pm 0.0241 ( $\mathrm{P}=0.004$ vs. Ben-Men-1), and $0.0411 \pm 0.0134$, respectively, which indicates that HMGN5 mRNA levels in IOMM-Lee and CH157 cell lines were significantly higher than those in Ben-Men-1 cells (Fig. 2A). Western blotting confirmed the same results (Fig. 2B). So IOMM-Lee and $\mathrm{CH} 157$ cells were used to perform the following experiments. Next, we established IOMM-Lee and $\mathrm{CH} 157$ cell lines stably expressing HMGN5-targeted siRNA. As shown in Fig. 2C and D, HMGN5 expression was significantly decreased on both mRNA and protein levels in IOMM-Lee and CH157 cells. We then measured the effects of HMGN5 expression on proliferation in IOMM-Lee and CH157 cells by using MTT assay and demonstrated that HMGN5 inhibition significantly suppressed the cell growth in both cell lines (Fig. 3A). In addition, flow cytometry revealed that the apoptosis rate was significantly enhanced in IOMM-Lee and CH157 cells underexpressing HMGN5 compared to control cells (Fig. 3B). These data indicate that knockdown of HMGN5 expression was able to inhibit cell proliferation and enhance apoptosis in meningioma cells.

Considering the increased rate of apoptosis in cells transfected with HMGN5-siRNA, we measured the expression of Bcl-2 and caspase-3 in IOMM-Lee and CH157 cells to explore the mechanism. Our data revealed that the mRNA and protein levels of Bcl-2 were significantly decreased in cells underexpressing HMGN5 compared to control cells (Fig. 3C). Conversely, the mRNA and protein expressions of cleavedcaspase-3 were significantly upregulated (Fig. 3C). These results suggest the antitumor effects of HMGN5 inhibition on 
A

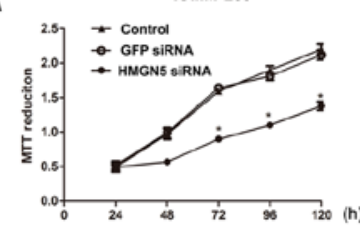

B

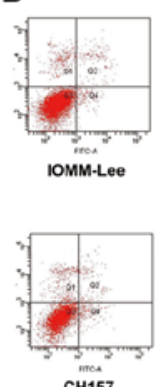

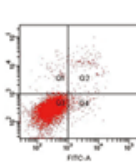

GFP SiRNA

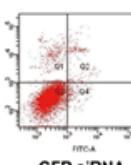

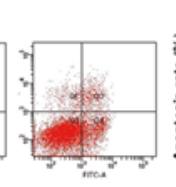

HMGN5 SIRNA

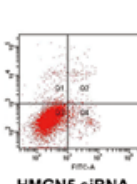

CH157
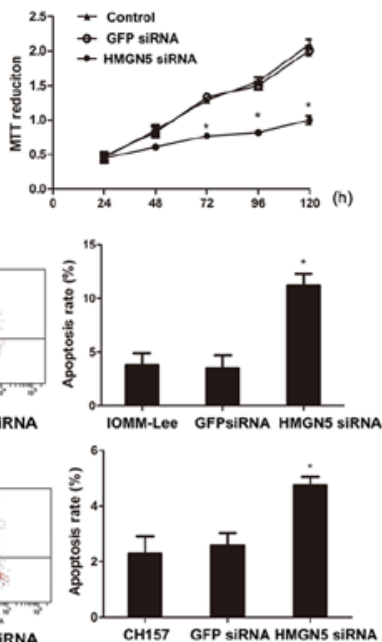

C
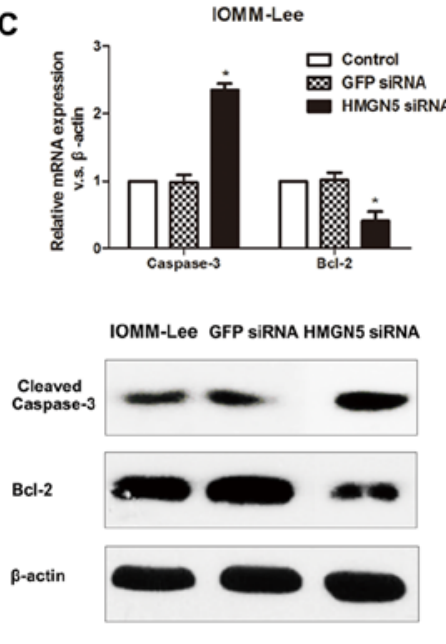

IOMM-Lee GFP SIRNA HMGN5 SIRNA

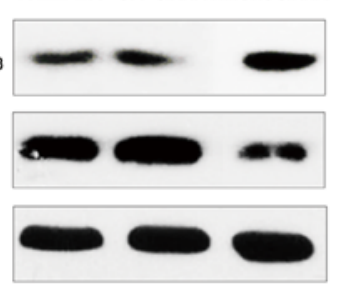

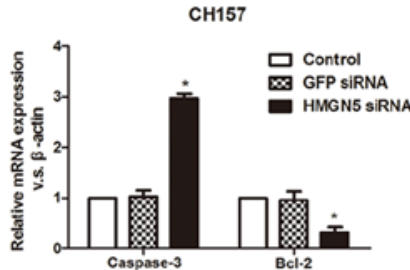

CH157 GFP SIRNA HMGN5 SIRNA

Figure 3. (A) Effect of HMGN5 knockdown on IOMM-Lee and CH157 cell proliferation as measured by the MTT assay. "P $<0.05$. (B) The number of apoptotic cells was significantly higher in IOMM-Lee and CH157 cells underexpressing HMGN5 as measured by PI staining and flow cytometry. "P< $<0.05$. (C) Bcl-2 and caspase-3 mRNA expression measured by qRT-PCR. Western blotting showed similar results.

A

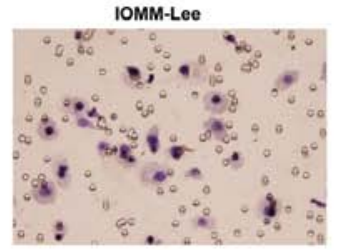

GFP SiRNA
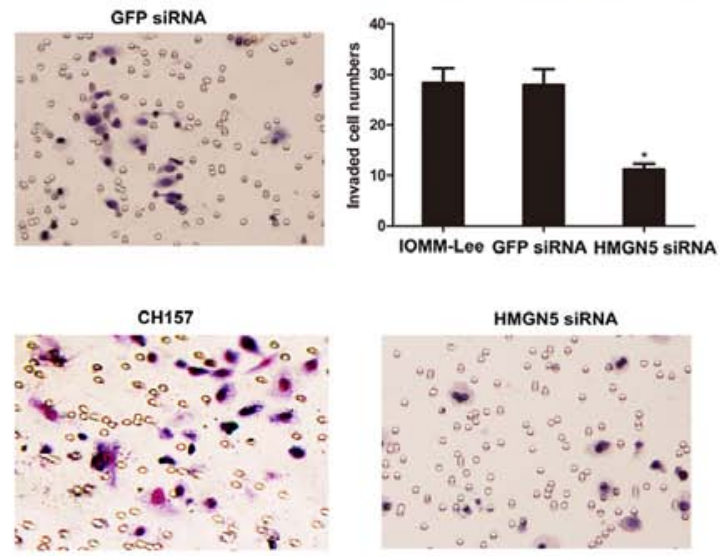

GFP siRNA

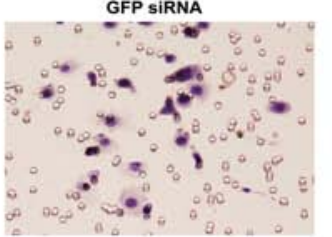

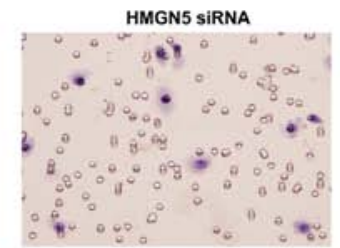
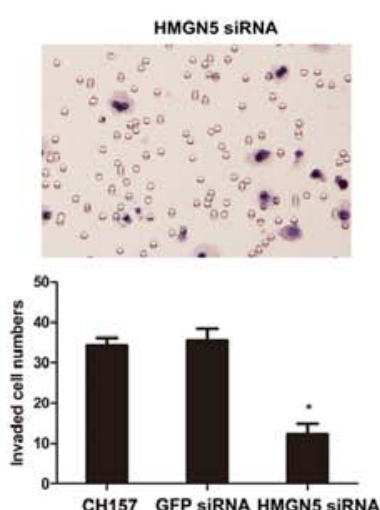

B

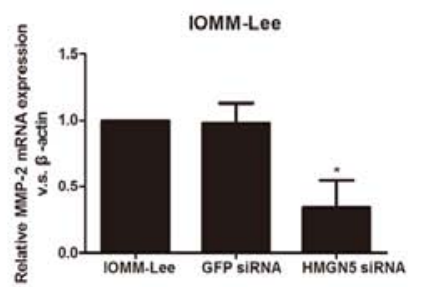

C

MMP-2

ß-actin

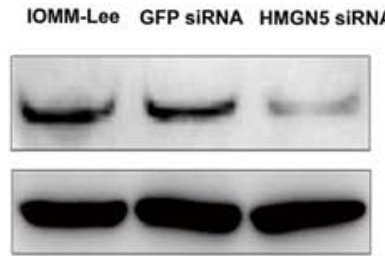

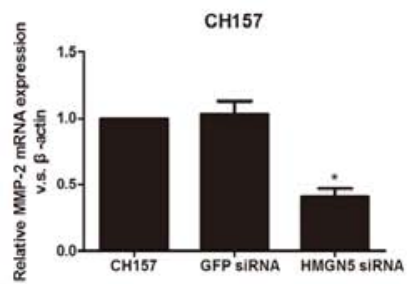

CH157 GFP SIRNA HMGN5 siRNA

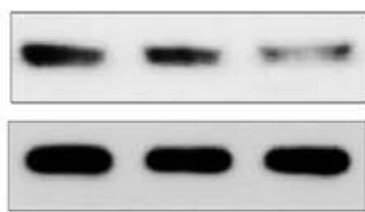

D

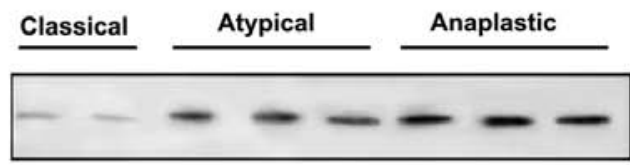

MMP-2

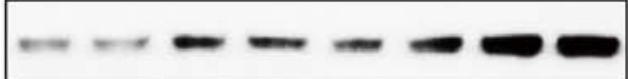

Actin

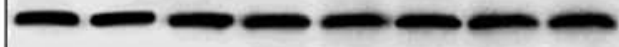

Figure 4. (A) HMGN5 inhibition in meningioma cells by siRNA transfection decreased invasion compared with GFP-siRNA and control cells in a Matrigel assay. "P<0.01. (B) qRT-PCR analysis demonstrates that MMP-2 mRNA was significantly suppressed in meningioma cells transfected with HMGN5-siRNA compared with control cells. "P<0.05. (C) Western blot analysis reveals reduced MMP-2 protein levels in meningioma cells transfected with HMGN5 siRNA compared to GFP-siRNA-transfected and control cells. (D) The protein levels of MMP-2 and HMGN5 in human meningioma specimens.

meningiomas are associated with enhanced caspase-3-dependence and suppressed the Bcl-2-induced apoptotic pathway.

Altered HMGN5 expression affects invasion in meningiomas. Invasion assay showed that knockdown of HMGN5 signifi- cantly suppressed the invasive activity of IOMM-Lee and CH157 cells, whereas the transfection of GFP-siRNA had no significant effects on the invasive activity of cells (Fig. 4A). Since the extracellular matrix metalloproteinases (MMPs) play a critical role in cell invasion processes, we measured 
A

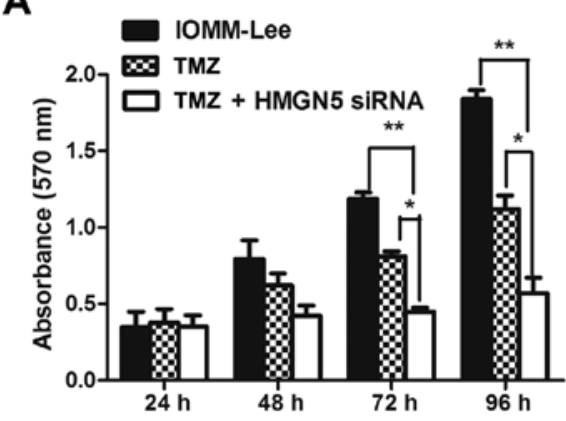

B
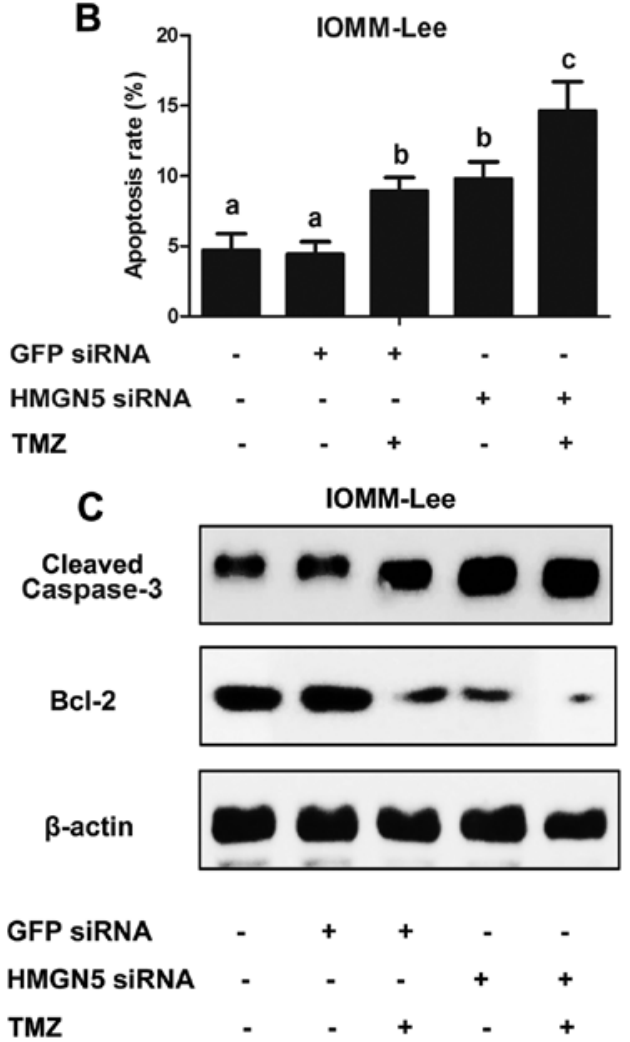
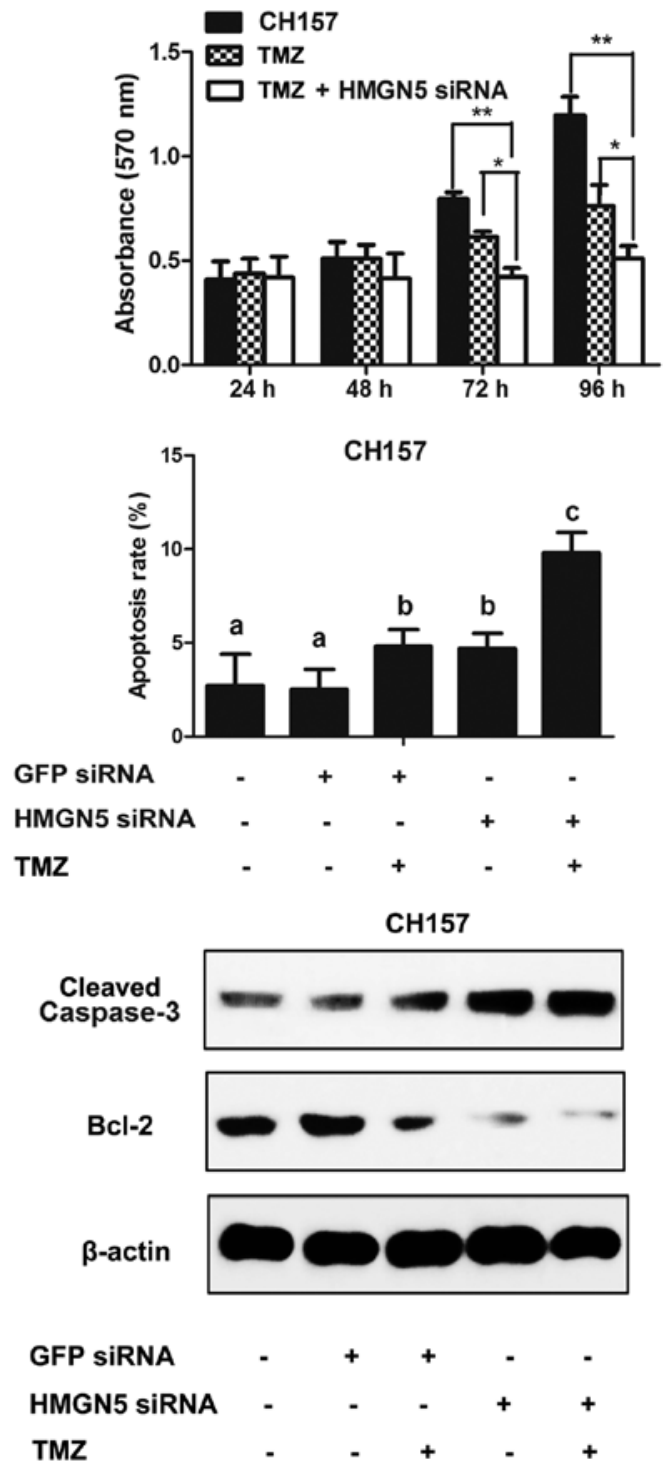

Figure 5. (A) HMGN5 knockdown causes increased growth inhibition by TMZ. MTT assays revealed that combined TMZ treatment and HMGN5 siRNA transfection led to significantly greater inhibition of proliferation than TMZ alone ( $\left(\mathrm{P}<0.05,{ }^{* *} \mathrm{P}<0.01\right)$. (B) Annexin $\mathrm{V} /$ propidium iodide staining shows that combined HMGN5 siRNA transfection and TMZ treatment resulted in a significant increase in apoptotic cell death compared with control cells. Values representing the mean $\pm \mathrm{SD}(\mathrm{n}=3)$ indicated with different letters, are significantly different. ${ }^{*} \mathrm{P}<0.05$. (C) Western blot analysis revealed reduced Bcl-2 and increased cleaved-caspase-3 protein levels in meningioma cells subjected to the combination siRNA transfection and TMZ treatment compared to cells treated with TMZ alone.

MMP-2 expression to further determine the mechanism of altered HMGN5 expression in meningioma cell invasion. We found downregulation of HMGN5 expression in IOMM-Lee and CH157 cells significantly suppressed MMP-2 expression on mRNA and protein levels (Fig. 4B and C). In addition, we further found MMP-2 protein expression was significantly increased in high grade meningiomas compared to low grade meningiomas by using western blotting (Fig. 4D). Moreover, western blot analysis indicated that MMP-2 expression associated positively with HMGN5 expression in human meningioma specimens (Fig. 4D). These data suggest that knockdown of HMGN5 results in the suppression of MMP-2 expression followed by a decrease of invasion.

HMGN5 regulates TMZ-induced cytotoxicity in meningiomas. We first measured the effects of HMGN5 expression on the TMZ cytotoxicity to IOMM-Lee and CH157 cells by using MTT assay. HMGN5 inhibition significantly increased TMZ-induced cytotoxicity in a time-dependent manner in both cell lines (Fig. 5A). We next examined the apoptosis rate of TMZ-induced apoptosis by flow cytometric analysis. As illustrated in Fig. 5B, HMGN5 siRNA alone could significantly enhance apoptosis compared to meningioma cells with cells transfected with GFP siRNA. When treated with $50 \mu \mathrm{M}$ TMZ, the combination of HMGN5 siRNA and TMZ significantly enhanced apoptosis of meningioma cells compared to those treated with TMZ alone (Fig. 5B). These results suggest that HMGN5 inhibition was able to sensitize TMZ-induced apoptosis.

We further found that HMGN5 inhibition significantly decreased Bcl-2 protein expression and increased cleavedcaspase-3 expression in both IOMM-Lee and CH157 cells 


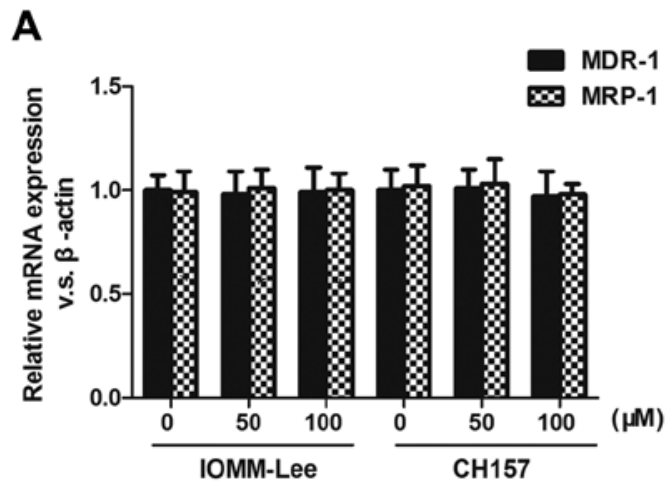

B

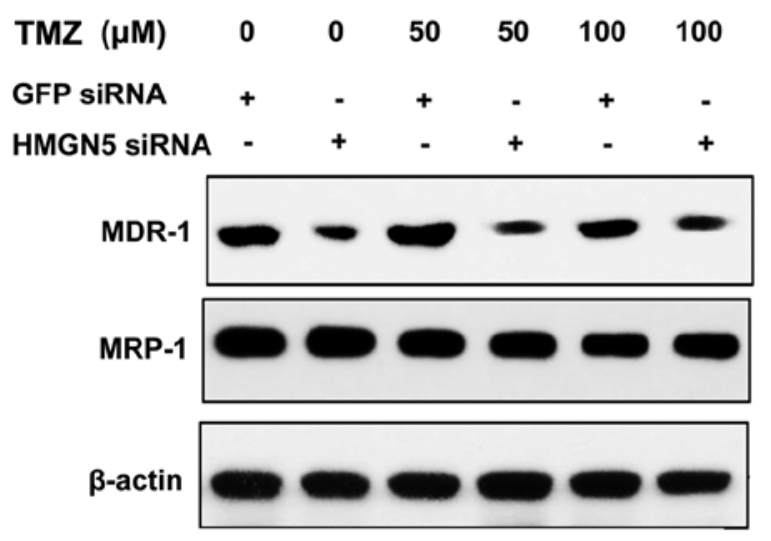

C

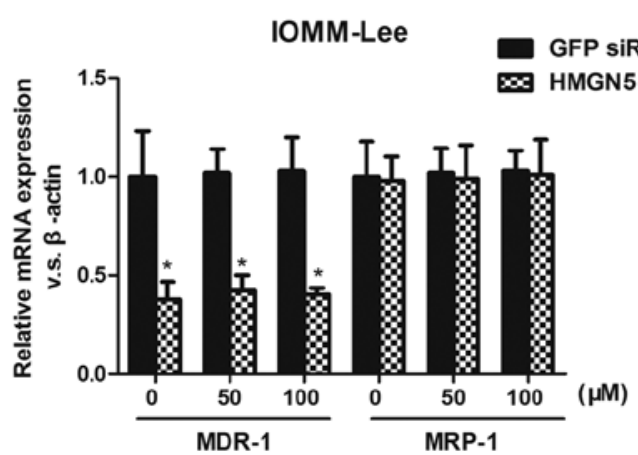

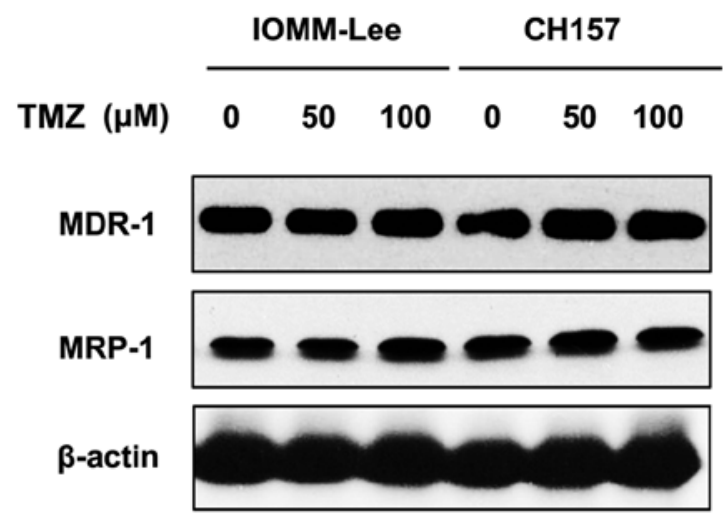

CH157
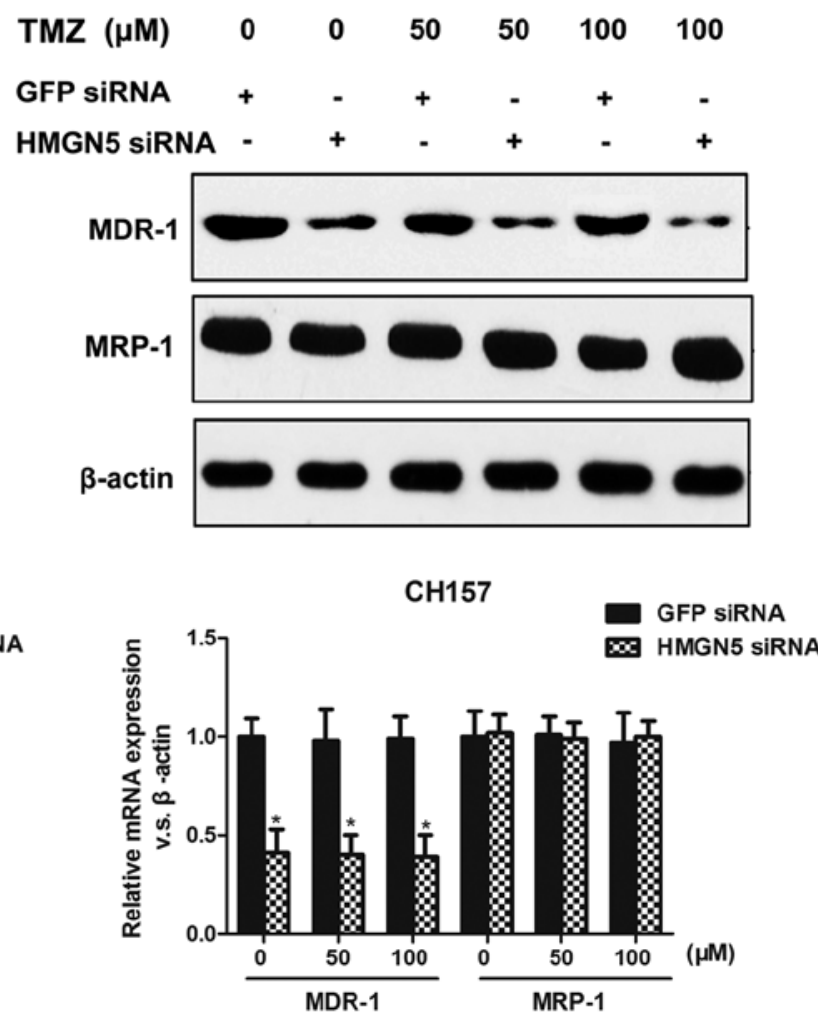

Figure 6. (A) qRT-PCR and western blotting revealed that different concentrations of TMZ ( 0,50 and $100 \mu \mathrm{M})$ failed to affect expression of MDR-1 and MRP-1 in both cell lines. (B) IOMM-Lee and CH157 cells were transfected with HMGN5 siRNA or GFP siRNA and then treated with indicated doses of TMZ (0, 50 and $100 \mu \mathrm{M}$ ) for $48 \mathrm{~h}$. Western blot analysis showed the alteration of MDR-1 protein expression. (C) qRT-PCR analysis revealed similar results. Relative mRNA ratios were normalized to $\beta$-actin. All data are mean $\pm \mathrm{SD},{ }^{*} \mathrm{P}<0.05$.

treated with TMZ when compared with cells treated with TMZ alone, which might explain the HMGN5 inhibition contributing to the improvement of chemoresitance (Fig. 5C). Thus, these results suggest that knockdown of HMGN5 expression sensitizes malignant meningioma cells to TMZ-induced apoptosis via regulating the expression of Bcl-2 and cleaved-caspase-3.

HMGN5 knockdown decreases MDR-1 expression without affecting MRP-1 expression. Considering that MDR-1 and MRP-1 proteins confer chemoresistance to tumor cells, we investigated whether HMGN5 alters TMZ sensitivity by mediating the expression of these proteins. We first confirmed that different doses of TMZ alone did not affect MDR-1 or MRP-1 expression (Fig. 6A). Next, we demonstrated that HMGN5 inhibition suppressed MDR-1 expression without affecting MRP-1 expression on protein levels in IOMM-Lee and CH157 cells treated with different doses of TMZ (Fig. 6B). Consistent with these western blot results, the mRNA expression of MDR-1 was significantly suppressed in IOMM-Lee and $\mathrm{CH} 157$ cells transfected with HMGN5-siRNA compared to controls, whereas MRP-1 expression did not change significantly between siRNA-transfected and GFP-siRNAtransfected meningioma cells (Fig. 6C). These data indicate that the increased susceptible to TMZ in meningioma cells underexpressing HMGN5 was associated with decreased MDR-1 expression and a concomitant suppression in MDR-1induced TMZ efflux. 


\section{Discussion}

Recent studies have suggested that HMGN5 is highly expressed in various cells derived from breast, uterine cancer, as well as gliomas (8-11), but little is known about its expression and functional role in meningiomas. Here, we measured HMGN5 expression levels in clinical meningioma specimens and revealed that HMGN5 expression was positively correlated with pathological grade and reccurence. Results from our study indicated that aberrantly high HMGN5 expression was a prognostic parameter for poor outcome. Immunohistochemical staining suggested that HMGN5 expression level could be used to predict the recurrence of meningiomas. In addition, we demonstrated a significant correlation between HMGN5 overexpression and elevated Ki-67 expression, a cellular proliferative marker, in human meningioma specimens. Furthermore, we showed that knockdown of HMGN5 expression in meningioma cells resulted in significantly lower cell numbers at 72,96 , and $120 \mathrm{~h}$ compared with control cells. Thus, our study provides both clinical and experimental evidence that HMGN5 may play an important role in proliferation and growth in meningioma cells.

We further found downregulation of HMGN5 enhanced apoptosis in IOMM-Lee and CH157 cells as indicated by the increase in the number of Annexin V/PI-positive cells measured by flow cytometry. The Bcl-2 family members are predominantly responsible for mediating the intrinsic apoptosis pathway (13). The most important member of this family is Bcl-2 itself $(14,15)$. Deregulation of apoptosis during tumor development can be caused by a disturbance in the homeostatic balance of the Bcl-2 family members (16). Caspases are a family of cysteine proteases with roles in apoptosis, inflammation, and development (17). Caspase-3 plays a critical role in evoking many of the defining biochemical and biophysical changes that occur during apoptosis (18). Previous studies have reported that elevated caspase-3 is associated with shorten overall survival in patients with malignant meningiomas (19). To explore the mechanisms of HMGN5-induced apoptosis in meningioma cells, we measured Bcl-2 and caspase-3 expression and suggested that reduced HMGN5 expression was correlated with increased effector caspase-3 expression and decreased anti-apoptotic Bcl-2 expression. Although there is no direct interaction of HMGN5 with either Bcl-2 or caspase-3, it is reasonable to speculate that HMGN5 plays a critical role in Bcl-2 and Cas-3 pathway. Additional studies are essential to fully describe HMGN5-related Bcl-2 and Cas-3 pathway in future.

MMPs play critical a role in promoting angiogenesis, tumor invasion, and tumor metastasis (20). Emerging evidence shows that overexpression of MMP-2 is correlated with the recurrence of intracranial meningiomas and predicts a poor prognosis in patients with advanced meningiomas $(21,22)$. It is also noteworthy that knockdown of MMP-2 could significantly suppress the invasive process in malignant brain tumors (23). Our results suggested that knockdown of HMGN5 significantly inhibited the invasiveness of meningioma cells. In addition, the mRNA and protein expression of MMP-2 in IOMM-Lee and CH157 cells was also significantly reduced after HMGN5 suppression. Thus, it is reasonable to speculate that high expression of HMGN5 could modulate matrix metalloproteinases signaling pathways, and by doing so, ultimately contributes to meningioma invasion.

$\mathrm{TMZ}$ is an oral alkylating chemotherapeutic agent that exerts antitumor effects by creating DNA damage in tumor cells. TMZ treatment has been reported to control treatmentresistant recurrent meningioma (24). Li et al demonstrated that the cytotoxic sensitivity to TMZ may be associated with its pro-apoptotic function (25). In this study, we found that that combined TMZ and HMGN5 inhibition showed enhanced inhibition on proliferation compared to TMZ treatment alone, indicating that HMGN5 is a promising therapeutic target to enhance the antitumor efficacy of TMZ. We further found that the combination of HMGN5 inhibition and TMZ significantly decreased Bcl-2 expression and increased caspase-3 expression, implying a functional interaction between HMGN5 and the apoptosis signaling pathways. A previous study demonstrated that upregulation of $\mathrm{Bcl}-2$ may protect against chemical agent-induced apoptosis in cancer cells (26). Thus, our data suggest a potential molecular mechanism for TMZ resistance in meningiomas that relies upon increased $\mathrm{Bcl}-2$ expression secondary to overactivation of the HMGN5 signaling pathway.

The ATP-binding cassette (ABC) superfamily of ATP-dependent efflux pumps is one of the largest protein families. Increased expression of ABC drug efflux transporters in tumors, which reduces intracellular doses of cytotoxic drugs, results in resistance to chemotherapy (27). Two major efflux pump families P-glycoprotein (MDR-1) and multidrug resistance associated proteins 1 (MRP-1) belong to the ABC superfamily. Importantly, TMZ has been reported to be a substrate of these ABC transporters (28). In this study, we found that knockdown of HMGN5 by siRNA significantly suppressed the MDR-1 expression without affecting MRP-1 expression in IOMM-Lee and CH157 cells. Moreover, the combination of HMGN5 siRNA and TMZ enhanced apoptosis when compared with cells treated with TMZ alone. This is in agreement with previous studies that MDR-1 may play a critical role in chemoresistance in malignant meningiomas (29). Taken together, we believed that HMGN5-dependent increase of MDR-1 may further suppress chemosensitivity, whereas decreased HMGN5 signaling could inhibit MDR-1 expression and drug efflux.

In conclusion, we demonstrated that knockdown of HMGN5 in IOMM-Lee and CH157 cells enhances apoptosis through increasing caspase- 3 and decreasing Bcl-2 expression, inhibits tumor invasiveness by suppressing MMP- 2 expression and increases chemosensitivity to TMZ via decreased MDR-1 expression without affecting expression of MRP-1. These results suggest that downregulation of HMGN5 expression may become an attractive strategy to treat human malignant meningioma in the future.

\section{References}

1. Fathi AR and Roelcke U: Meningioma. Curr Neurol Neurosci Rep 13: 337, 2013.

2. Dubel GJ, Ahn SH and Soares GM: Contemporary endovascular embolotherapy for meningioma. Semin Intervent Radiol 30: 263-277, 2013.

3. Walcott BP, Nahed BV, Brastianos PK and Loeffler JS: Radiation Treatment for WHO Grade II and III meningiomas. Front Oncol 3: 227, 2013. 
4. Saraf S, McCarthy BJ and Villano JL: Update on meningiomas. Oncologist 16: 1604-1613, 2011.

5. Rochman M, Malicet $C$ and Bustin M: HMGN5/NSBP1: A new member of the HMGN protein family that affects chromatin structure and function. Biochim Biophys Acta 1799: 86-92, 2010

6. Shirakawa H, Landsman D, Postnikov YV and Bustin M NBP-45, a novel nucleosomal binding protein with a tissuespecific and developmentally regulated expression. J Biol Chem 275: 6368-6374, 2000

7. Rochman M, Postnikov Y, Correll S, Malicet C, Wincovitch S, Karpova TS, McNally JG, Wu X, Bubunenko NA, Grigoryev S, et al: The interaction of NSBP1/HMGN5 with nucleosomes in euchromatin counteracts linker histone-mediated chromatin compaction and modulates transcription. Mol Cell 35: 642-656, 2009 .

8. Ji SQ, Yao L, Zhang XY, Li XS and Zhou LQ: Knockdown of the nucleosome binding protein 1 inhibits the growth and invasion of clear cell renal cell carcinoma cells in vitro and in vivo. J Exp Clin Cancer Res 31: 22, 2012.

9. Wahafu W, He ZS, Zhang XY, Zhang CJ, Yao K, Hao H, Song G, He Q, Li XS and Zhou LQ: The nucleosome binding protein NSBP1 is highly expressed in human bladder cancer and promotes the proliferation and invasion of bladder cancer cells. Tumour Biol 32: 931-939, 2011.

10. Jiang N, Zhou LQ and Zhang XY: Downregulation of the nucleosome-binding protein 1 (NSBP1) gene can inhibit the in vitro and in vivo proliferation of prostate cancer cells. Asian J Androl 12: 709-717, 2010.

11. Qu J, Yan R, Chen J, Xu T, Zhou J, Wang M, Chen C, Yan Y and Lu Y: HMGN5: A potential oncogene in gliomas. J Neurooncol 104: 729-736, 2011.

12. Wang Q, Wang JY, Zhang XP, Lv ZW, Fu D, Lu YC, Hu GH, Luo C and Chen JX: RLIP76 is overexpressed in human glioblastomas and is required for proliferation, tumorigenesis and suppression of apoptosis. Carcinogenesis 34: 916-926, 2013.

13. Martinou JC and Youle RJ: Mitochondria in apoptosis: Bcl-2 family members and mitochondrial dynamics. Dev Cell 21: 92-101, 2011.

14. Soane L and Fiskum G: Inhibition of mitochondrial neural cell death pathways by protein transduction of $\mathrm{Bcl}-2$ family proteins. J Bioenerg Biomembr 37: 179-190, 2005.

15. Basu A, DuBois G and Haldar S: Posttranslational modifications of Bcl2 family members - a potential therapeutic target for human malignancy. Front Biosci 11: 1508-1521, 2006.

16. Zeestraten EC, Benard A, Reimers MS, Schouten PC, Liefers GJ, van de Velde CJ and Kuppen PJ: The prognostic value of the apoptosis pathway in colorectal cancer: A review of the literature on biomarkers identified by immunohistochemistry. Biomark Cancer 5: 13-29, 2013.
17. Murray $\mathbf{J}$ and Renslo AR: Modulating caspase activity: Beyond the active site. Curr Opin Struct Biol 23: 812-819, 2013.

18. McCracken JM and Allen LA: Regulation of human neutrophil apoptosis and lifespan in health and disease. J Cell Death 7: 15-23, 2014.

19. Vranic A: Caspase-3 and survivin expression in primary atypical and malignant meningiomas. ISRN Neurosci 2013: 626290, 2013.

20. Shiraga M, Yano S, Yamamoto A, Ogawa H, Goto H, Miki T, Miki K, Zhang H and Sone S: Organ heterogeneity of hostderived matrix metalloproteinase expression and its involvement in multiple-organ metastasis by lung cancer cell lines. Cancer Res 62: 5967-5973, 2002.

21. Mandara MT, Pavone S, Mandrioli L, Bettini G, Falzone C and Baroni M: Matrix metalloproteinase-2 and matrix metalloproteinase-9 expression in canine and feline meningioma. Vet Pathol 46: 836-845, 2009.

22. Okada M, Miyake K, Matsumoto Y, Kawai N, Kunishio K and Nagao S: Matrix metalloproteinase-2 and matrix metalloproteinase- 9 expressions correlate with the recurrence of intracranial meningiomas. J Neurooncol 66: 29-37, 2004.

23. Kast RE and Halatsch ME: Matrix metalloproteinase-2 and -9 in glioblastoma: A trio of old drugs - captopril, disulfiram and nelfinavir - are inhibitors with potential as adjunctive treatments in glioblastoma. Arch Med Res 43: 243-247, 2012.

24. Chamberlain MC, Tsao-Wei DD and Groshen S: Temozolomide for treatment-resistant recurrent meningioma. Neurology 62 : 1210-1212, 2004

25. Li G, Zhang H, Liu Y, Kong L, Guo Q and Jin F: Effect of temozolomide on livin and caspase-3 in U251 glioma stem cells. Exp Ther Med 9: 744-750, 2015.

26. Akay C, Thomas C III and Gazitt Y: Arsenic trioxide and paclitaxel induce apoptosis by different mechanisms. Cell Cycle 3: 324-334, 2004

27. Wang Q, Qian J, Wang J, Luo C, Chen J, Hu G and Lu Y: Knockdown of RLIP76 expression by RNA interference inhibits invasion, induces cell cycle arrest, and increases chemosensitivity to the anticancer drug temozolomide in glioma cells. J Neurooncol 112: 73-82, 2013.

28. Schaich M, Kestel L, Pfirrmann M, Robel K, Illmer T, Kramer M, Dill C, Ehninger G, Schackert G and Krex D.: A MDR1 (ABCB1) gene single nucleotide polymorphism predicts outcome of temozolomide treatment in glioblastoma patients. Ann Oncol 20: 175-181, 2009.

29. Andersson U, Malmer B, Bergenheim AT, Brännström T and Henriksson R: Heterogeneity in the expression of markers for drug resistance in brain tumors. Clin Neuropathol 23: 21-27, 2004. 